\title{
Seven octonary quadratic forms
}

by

Ayşe Alaca, Şaban Alaca and Kenneth S. Williams (Ottawa)

1. Introduction. Let $\mathbb{N}, \mathbb{N}_{0}, \mathbb{Z}$ and $\mathbb{C}$ denote the sets of positive integers, nonnegative integers, integers and complex numbers respectively. For $k, a_{1}, \ldots, a_{k} \in \mathbb{N}$ and $n \in \mathbb{N}_{0}$, we define

$$
N\left(a_{1}, \ldots, a_{k} ; n\right):=\operatorname{card}\left\{\left(x_{1}, \ldots, x_{k}\right) \in \mathbb{Z}^{k} \mid n=a_{1} x_{1}^{2}+\cdots+a_{k} x_{k}^{2}\right\} .
$$

As $N\left(a_{1}, \ldots, a_{k} ; n\right)$ remains invariant under a permutation of $a_{1}, \ldots, a_{k}$, we may suppose that

$$
a_{1} \leq \cdots \leq a_{k}
$$

Clearly,

$$
N\left(a_{1}, \ldots, a_{k} ; 0\right)=1 .
$$

If $l$ of $a_{1}, \ldots, a_{k}$ are equal, say

$$
a_{i}=a_{i+1}=\cdots=a_{i+l-1}=a,
$$

we indicate this in $N\left(a_{1}, \ldots, a_{k} ; n\right)$ by writing $a^{l}$ for $a_{i}, a_{i+1}, \ldots, a_{i+l-1}$. For $k \in \mathbb{N}$ the sum of divisors function $\sigma_{k}(n)$ is defined by

$$
\sigma_{k}(n):= \begin{cases}\sum_{d \in \mathbb{N}} d^{k} & \text { if } n \in \mathbb{N}, \\ d \mid n & \text { if } n \notin \mathbb{N} .\end{cases}
$$

We write $\sigma(n)$ for $\sigma_{1}(n)$.

The authors and M. F. Lemire have recently proved formulae for $N\left(1^{i}, 4^{4-i} ; n\right)$ for $i \in\{1,2,3,4\}$ and all $n \in \mathbb{N}$ in terms of $\sigma(n), \sigma(n / 2)$, $\sigma(n / 4), \sigma(n / 8)$ and $\sigma(n / 16)$ [1, Theorems 1.6, 1.7, 1.11 and 1.18]. The origins of these formulae are given in [1, pp. 284-286].

2000 Mathematics Subject Classification: Primary 11E25.

Key words and phrases: octonary quadratic forms, convolution sums, sum of divisors function.

The research of the second and third authors was supported by research grants from the Natural Sciences and Engineering Research Council of Canada. 
Proposition 1.1. Let $n \in \mathbb{N}$. Then

$$
\begin{aligned}
N\left(1^{4} ; n\right)= & 8 \sigma(n)-32 \sigma(n / 4), \\
N\left(1^{3}, 4 ; n\right)= & \left(4+2\left(\frac{-4}{n}\right)\right) \sigma(n)-20 \sigma(n / 4) \\
& +24 \sigma(n / 8)-32 \sigma(n / 16), \\
N\left(1^{2}, 4^{2} ; n\right)= & \left(2+2\left(\frac{-4}{n}\right)\right) \sigma(n)-2 \sigma(n / 2) \\
& +8 \sigma(n / 8)-32 \sigma(n / 16), \\
N\left(1,4^{3} ; n\right)= & \left(1+\left(\frac{-4}{n}\right)\right) \sigma(n)-3 \sigma(n / 2) \\
& +10 \sigma(n / 4)-32 \sigma(n / 16),
\end{aligned}
$$

where $\left(\frac{-4}{n}\right)$ is the Legendre-Jacobi-Kronecker symbol for discriminant -4 , that is,

$$
\left(\frac{-4}{n}\right)= \begin{cases}+1 & \text { if } n \equiv 1(\bmod 4), \\ -1 & \text { if } n \equiv 3(\bmod 4), \\ 0 & \text { if } n \equiv 0(\bmod 2) .\end{cases}
$$

Proof. See [1, pp. 296, 297, 298, 303].

Definition 1.1. For $k, n \in \mathbb{N}$ we define

$$
W_{k}(n):=\sum_{\substack{m \in \mathbb{N} \\ m<n / k}} \sigma(m) \sigma(n-k m) .
$$

In recent years the convolution sums $W_{k}(n)$ have been evaluated explicitly for certain values of $k$ and all $n \in \mathbb{N}$. We require the evaluations for $k=1$ [6, eq. (3.10), p. 236], $k=2$ [6, Theorem 2, p. 247], $k=4$ [6, Theorem 4, p. 249], $k=8$ [7, Theorem 1, p. 388], and $k=16$ [2, Theorem 1.1, p. 4].

Proposition 1.2. Let $n \in \mathbb{N}$. Then

$$
\begin{aligned}
& W_{1}(n)=\frac{5}{12} \sigma_{3}(n)+\left(\frac{1}{12}-\frac{n}{2}\right) \sigma(n) . \\
& W_{2}(n)=\frac{1}{12} \sigma_{3}(n)+\frac{1}{3} \sigma_{3}(n / 2)+\left(\frac{1}{24}-\frac{n}{8}\right) \sigma(n) \\
& +\left(\frac{1}{24}-\frac{n}{4}\right) \sigma(n / 2) \text {. } \\
& \text { (iii) } \quad W_{4}(n)=\frac{1}{48} \sigma_{3}(n)+\frac{1}{16} \sigma_{3}(n / 2)+\frac{1}{3} \sigma_{3}(n / 4) \\
& +\left(\frac{1}{24}-\frac{n}{16}\right) \sigma(n)+\left(\frac{1}{24}-\frac{n}{4}\right) \sigma(n / 4) \text {. }
\end{aligned}
$$


(iv) $\quad W_{8}(n)=\frac{1}{192} \sigma_{3}(n)+\frac{1}{64} \sigma_{3}(n / 2)+\frac{1}{16} \sigma_{3}(n / 4)+\frac{1}{3} \sigma_{3}(n / 8)$

$$
+\left(\frac{1}{24}-\frac{n}{32}\right) \sigma(n)+\left(\frac{1}{24}-\frac{n}{4}\right) \sigma(n / 8)-\frac{1}{64} c_{8}(n),
$$

where the integers $c_{8}(n)(n \in \mathbb{N})$ are given by

$$
\sum_{n=1}^{\infty} c_{8}(n) q^{n}=q \prod_{n=1}^{\infty}\left(1-q^{2 n}\right)^{4}\left(1-q^{4 n}\right)^{4} .
$$

(v) $W_{16}(n)=\frac{1}{768} \sigma_{3}(n)+\frac{1}{256} \sigma_{3}(n / 2)$

$$
\begin{aligned}
& +\frac{1}{64} \sigma_{3}(n / 4)+\frac{1}{16} \sigma_{3}(n / 8)+\frac{1}{3} \sigma_{3}(n / 16) \\
& +\left(\frac{1}{24}-\frac{n}{64}\right) \sigma(n)+\left(\frac{1}{24}-\frac{n}{4}\right) \sigma(n / 16)-\frac{7}{256} c_{16}(n),
\end{aligned}
$$

where the rational numbers $c_{16}(n)(n \in \mathbb{N})$ are given by

$$
\begin{aligned}
\sum_{n=1}^{\infty} c_{16}(n) q^{n}= & \frac{1}{32} A_{1}(q)+\frac{3}{112} A_{2}(q)+\frac{1}{224} A_{3}(q) \\
& -\frac{1}{32} A_{5}(q)-\frac{3}{112} A_{6}(q)-\frac{1}{224} A_{7}(q)
\end{aligned}
$$

with

$$
A_{k}(q):=\prod_{n=1}^{\infty}\left(1+q^{n}\right)^{24-4 k}\left(1-q^{n}\right)^{8}\left(1-q^{4 n-2}\right)^{16-2 k} .
$$

We remark that for $n \in \mathbb{N}$,

$$
c_{8}(n)=0 \quad \text { if } n \equiv 0(\bmod 2)
$$

(see [7, p. 388]), and

$$
7 c_{16}(n) \in \mathbb{Z}
$$

(see [2, eq. (1.6), p. 4]).

Definition 1.2. For $a \in \mathbb{Z}$ and $m, n \in \mathbb{N}$ we define

$$
S_{a, m}(n):=\sum_{\substack{l=1 \\ l \equiv a(\bmod m)}}^{n-1} \sigma(l) \sigma(n-l) .
$$

Clearly,

$$
S_{a, m}(n)=S_{b, m}(n) \quad \text { if } a \equiv b(\bmod m),
$$


and

$$
\sum_{a=0}^{m-1} S_{a, m}(n)=W_{1}(n) .
$$

These sums were introduced in $[6$, p. 255]. We require the evaluation of $S_{a, 4}(n)$ given in [3, Theorem 1.1].

Proposition 1.3. Let $n \in \mathbb{N}$.

(i) If $n \equiv 0(\bmod 4)$ then

$$
\begin{aligned}
& S_{0,4}(n)=\frac{29}{192} \sigma_{3}(n)+\frac{17}{64} \sigma_{3}(n / 2)+\left(\frac{1}{12}-\frac{n}{2}\right) \sigma(n), \\
& S_{1,4}(n)=\frac{1}{16} \sigma_{3}(n)-\frac{1}{16} \sigma_{3}(n / 2), \\
& S_{2,4}(n)=\frac{9}{64} \sigma_{3}(n)-\frac{9}{64} \sigma_{3}(n / 2), \\
& S_{3,4}(n)=\frac{1}{16} \sigma_{3}(n)-\frac{1}{16} \sigma_{3}(n / 2) .
\end{aligned}
$$

(ii) If $n \equiv 1(\bmod 4)$ then

$$
\begin{aligned}
& S_{0,4}(n)=\frac{11}{96} \sigma_{3}(n)+\left(\frac{1}{24}-\frac{n}{4}\right) \sigma(n)+\frac{3}{32} c_{8}(n), \\
& S_{1,4}(n)=\frac{11}{96} \sigma_{3}(n)+\left(\frac{1}{24}-\frac{n}{4}\right) \sigma(n)+\frac{3}{32} c_{8}(n), \\
& S_{2,4}(n)=\frac{3}{32} \sigma_{3}(n)-\frac{3}{32} c_{8}(n), \\
& S_{3,4}(n)=\frac{3}{32} \sigma_{3}(n)-\frac{3}{32} c_{8}(n) .
\end{aligned}
$$

(iii) If $n \equiv 2(\bmod 4)$ then

$$
\begin{aligned}
& S_{0,4}(n)=\frac{11}{72} \sigma_{3}(n)+\left(\frac{1}{24}-\frac{n}{4}\right) \sigma(n), \\
& S_{1,4}(n)=\frac{1}{18} \sigma_{3}(n)+\frac{1}{2} c_{8}(n / 2), \\
& S_{2,4}(n)=\frac{11}{72} \sigma_{3}(n)+\left(\frac{1}{24}-\frac{n}{4}\right) \sigma(n), \\
& S_{3,4}(n)=\frac{1}{18} \sigma_{3}(n)-\frac{1}{2} c_{8}(n / 2) .
\end{aligned}
$$

(iv) If $n \equiv 3(\bmod 4)$ then

$$
S_{0,4}(n)=\frac{11}{96} \sigma_{3}(n)+\left(\frac{1}{24}-\frac{n}{4}\right) \sigma(n)+\frac{3}{32} c_{8}(n),
$$




$$
\begin{aligned}
& S_{1,4}(n)=\frac{3}{32} \sigma_{3}(n)-\frac{3}{32} c_{8}(n), \\
& S_{2,4}(n)=\frac{3}{32} \sigma_{3}(n)-\frac{3}{32} c_{8}(n), \\
& S_{3,4}(n)=\frac{11}{96} \sigma_{3}(n)+\left(\frac{1}{24}-\frac{n}{4}\right) \sigma(n)+\frac{3}{32} c_{8}(n) .
\end{aligned}
$$

In this paper we use Propositions 1.1, 1.2 and 1.3 to determine $N\left(1^{i}, 4^{8-i} ; n\right)$ for $i \in\{1,2,3,4,5,6,7\}$ and all $n \in \mathbb{N}$. We prove

Theorem 1.1. Let $n \in \mathbb{N}$. Then

$$
\begin{aligned}
N\left(1^{7}, 4 ; n\right)= & \left(8-\left(\frac{-4}{n}\right)\right) \sigma_{3}(n)-16 \sigma_{3}(n / 2)+136 \sigma_{3}(n / 4) \\
& -144 \sigma_{3}(n / 8)+256 \sigma_{3}(n / 16) \\
& +7\left(\frac{-4}{n}\right) c_{8}(n)+28 c_{8}(n / 2) .
\end{aligned}
$$

(ii) $\quad N\left(1^{6}, 4^{2} ; n\right)=\left(4-\left(\frac{-4}{n}\right)\right) \sigma_{3}(n)-4 \sigma_{3}(n / 2)-16 \sigma_{3}(n / 8)$

$$
+256 \sigma_{3}(n / 16)+\left(2+7\left(\frac{-4}{n}\right)\right) c_{8}(n)+28 c_{8}(n / 2) .
$$

(iii) $\quad N\left(1^{5}, 4^{3} ; n\right)=\left(2-\frac{1}{2}\left(\frac{-4}{n}\right)\right) \sigma_{3}(n)+2 \sigma_{3}(n / 2)-68 \sigma_{3}(n / 4)$

$$
\begin{aligned}
& +48 \sigma_{3}(n / 8)+256 \sigma_{3}(n / 16) \\
& +\left(3+\frac{11}{2}\left(\frac{-4}{n}\right)\right) c_{8}(n)+20 c_{8}(n / 2) .
\end{aligned}
$$

(iv) $\quad N\left(1^{4}, 4^{4} ; n\right)=\sigma_{3}(n)+3 \sigma_{3}(n / 2)-68 \sigma_{3}(n / 4)+48 \sigma_{3}(n / 8)$

$$
+256 \sigma_{3}(n / 16)+\left(3+4\left(\frac{-4}{n}\right)\right) c_{8}(n)+12 c_{8}(n / 2) .
$$

(v) $\quad N\left(1^{3}, 4^{5} ; n\right)=\left(\frac{1}{2}+\frac{1}{4}\left(\frac{-4}{n}\right)\right) \sigma_{3}(n)+\frac{3}{2} \sigma_{3}(n / 2)-34 \sigma_{3}(n / 4)$

$$
\begin{aligned}
& +16 \sigma_{3}(n / 8)+256 \sigma_{3}(n / 16) \\
& +\left(\frac{5}{2}+\frac{11}{4}\left(\frac{-4}{n}\right)\right) c_{8}(n)+6 c_{8}(n / 2) .
\end{aligned}
$$

(vi) $\quad N\left(1^{2}, 4^{6} ; n\right)=\left(\frac{1}{4}+\frac{1}{4}\left(\frac{-4}{n}\right)\right) \sigma_{3}(n)-\frac{1}{4} \sigma_{3}(n / 2)-16 \sigma_{3}(n / 8)$

$$
+256 \sigma_{3}(n / 16)+\left(\frac{7}{4}+\frac{7}{4}\left(\frac{-4}{n}\right)\right) c_{8}(n)+2 c_{8}(n / 2) .
$$


(vii)

$$
\begin{aligned}
N\left(1,4^{7} ; n\right)= & \left(\frac{1}{8}+\frac{1}{8}\left(\frac{-4}{n}\right)\right) \sigma_{3}(n)-\frac{9}{8} \sigma_{3}(n / 2)+17 \sigma_{3}(n / 4) \\
& -32 \sigma_{3}(n / 8)+256 \sigma_{3}(n / 16)+\left(\frac{7}{8}+\frac{7}{8}\left(\frac{-4}{n}\right)\right) c_{8}(n) .
\end{aligned}
$$

Part (iv) of Theorem 1.1 was proved in [2, Theorem 1.2, p. 4] in terms of $c_{16}$ rather than $c_{8}$.

2. Some preliminary results. The following sums will be needed in the proof of Theorem 1.1.

Definition 2.1. For $r, s \in \mathbb{N}_{0}$ and $n \in \mathbb{N}$, we define

$$
X\left(2^{r}, 2^{s} ; n\right):=\sum_{m=1}^{n-1} \sigma\left(m / 2^{r}\right) \sigma\left((n-m) / 2^{s}\right) .
$$

Clearly,

$$
X\left(2^{r}, 2^{s} ; n\right)=X\left(2^{s}, 2^{r} ; n\right) .
$$

Proposition 2.1. Let $r, s \in \mathbb{N}_{0}$ and $n \in \mathbb{N}$. Then

$$
X\left(2^{r}, 2^{s} ; n\right)= \begin{cases}W_{2^{r-s}}\left(n / 2^{s}\right) & \text { if } r \geq s, \\ W_{2^{s-r}}\left(n / 2^{r}\right) & \text { if } r \leq s .\end{cases}
$$

Proof. If $r \geq s$ then

$$
X\left(2^{r}, 2^{s} ; n\right)=\sum_{\substack{m \in \mathbb{N} \\ m<n / 2^{r}}} \sigma(m) \sigma\left(\frac{n}{2^{s}}-2^{r-s} m\right)=W_{2^{r-s}}\left(n / 2^{s}\right) .
$$

If $r \leq s$ then

$$
X\left(2^{r}, 2^{s} ; n\right)=X\left(2^{s}, 2^{r} ; n\right)=W_{2^{s-r}}\left(n / 2^{r}\right) .
$$

Proposition 2.2. For $n \in \mathbb{N}$,

$$
\begin{aligned}
& \sum_{m=1}^{n-1}\left(\frac{-4}{m}\right) \sigma(m) \sigma(n-m) \\
& \quad=\left(\frac{-4}{n}\right)\left(\frac{1}{48} \sigma_{3}(n)+\left(\frac{1}{24}-\frac{n}{4}\right) \sigma(n)+\frac{3}{16} c_{8}(n)\right)+c_{8}(n / 2) .
\end{aligned}
$$


Proof. We have

$$
\begin{aligned}
\sum_{m=1}^{n-1}\left(\frac{-4}{m}\right) & \sigma(m) \sigma(n-m) \\
& =\sum_{\substack{m=1 \\
m \equiv 1(\bmod 4)}}^{n-1} \sigma(m) \sigma(n-m)-\sum_{\substack{m=1 \\
m \equiv 3(\bmod 4)}}^{n-1} \sigma(m) \sigma(n-m) \\
& =S_{1,4}(n)-S_{3,4}(n)
\end{aligned}
$$

by Definition 1.2, and the asserted result follows from Proposition 1.3.

Proposition 2.3. For $n \in \mathbb{N}$,

$$
\sum_{m=1}^{n-1}\left(\frac{-4}{m}\right) \sigma(m) \sigma((n-m) / 2)=\left(\frac{-4}{n}\right)\left(-W_{2}(n)+6 W_{4}(n)-4 W_{8}(n)\right) .
$$

Proof. We have

$$
\begin{aligned}
\sum_{m=1}^{n-1}\left(\frac{-4}{m}\right) \sigma(m) \sigma((n-m) / 2)=\sum_{\substack{l \in \mathbb{N} \\
l<n / 2}}\left(\frac{-4}{n-2 l}\right) \sigma(n-2 l) \sigma(l) \\
=\sum_{\substack{l \in \mathbb{N} \\
l<n / 4}}\left(\frac{-4}{n-4 l}\right) \sigma(2 l) \sigma(n-4 l) \\
\quad+\sum_{\substack{l \in \mathbb{N} \\
l<(n+2) / 4}}\left(\frac{-4}{n-2(2 l-1)}\right) \sigma(2 l-1) \sigma(n-2(2 l-1)) \\
=\left(\frac{-4}{n}\right) \sum_{\substack{l \in \mathbb{N} \\
l<n / 4}} \sigma(2 l) \sigma(n-4 l) \\
\quad+\left(\frac{-4}{n+2}\right) \sum_{\substack{l \in \mathbb{N} \\
l<(n+2) / 4}} \sigma(2 l-1) \sigma(n-2(2 l-1)) \\
=\left(\frac{-4}{n}\right) \sum_{\substack{l \in \mathbb{N} \\
l<n / 4}} \sigma(2 l) \sigma(n-4 l) \\
\quad-\left(\frac{-4}{n}\right)\left(\sum_{\substack{l \in \mathbb{N} \\
l<n / 2}} \sigma(l) \sigma(n-2 l)-\sum_{\substack{l \in \mathbb{N} \\
l<n / 4}} \sigma(2 l) \sigma(n-4 l)\right)
\end{aligned}
$$




$$
\begin{aligned}
& =-\left(\frac{-4}{n}\right) \sum_{\substack{l \in \mathbb{N} \\
l<n / 2}} \sigma(l) \sigma(n-2 l)+2\left(\frac{-4}{n}\right) \sum_{\substack{l \in \mathbb{N} \\
l<n / 4}} \sigma(2 l) \sigma(n-4 l) \\
& =-\left(\frac{-4}{n}\right) \sum_{\substack{l \in \mathbb{N} \\
l<n / 2}} \sigma(l) \sigma(n-2 l)+2\left(\frac{-4}{n}\right) \sum_{\substack{l \in \mathbb{N} \\
l<n / 4}}(3 \sigma(l)-2 \sigma(l / 2)) \sigma(n-4 l) \\
& =\left(\frac{-4}{n}\right)\left(-W_{2}(n)+6 W_{4}(n)-4 W_{8}(n)\right),
\end{aligned}
$$

as asserted.

Proposition 2.4. For $k, n \in \mathbb{N}$ with $k \geq 2$,

$$
\sum_{m=1}^{n-1}\left(\frac{-4}{m}\right) \sigma(m) \sigma\left((n-m) / 2^{k}\right)=\left(\frac{-4}{n}\right) W_{2^{k}}(n) .
$$

Proof. As $k \geq 2$ we have

$$
\left(\frac{-4}{n-2^{k l}}\right)=\left(\frac{-4}{n}\right)
$$

and so

$$
\begin{aligned}
\sum_{m=1}^{n-1}\left(\frac{-4}{m}\right) \sigma(m) \sigma\left((n-m) / 2^{k}\right) & =\sum_{\substack{l \in \mathbb{N} \\
l<n / 2^{k}}}\left(\frac{-4}{n-2^{k} l}\right) \sigma\left(n-2^{k} l\right) \sigma(l) \\
& =\left(\frac{-4}{n}\right) \sum_{\substack{l \in \mathbb{N} \\
l<n / 2^{k}}} \sigma(l) \sigma\left(n-2^{k} l\right)=\left(\frac{-4}{n}\right) W_{2^{k}}(n)
\end{aligned}
$$

by Definition 1.1 .

3. The relationship between $c_{8}(n)$ and $c_{16}(n)$. Let $q$ be a complex variable with $|q|<1$. As in $[4$, p. 6$]$ we set

$$
\varphi(q):=\sum_{n=-\infty}^{\infty} q^{n^{2}}
$$

The infinite product representations of $\varphi(q)$ and $\varphi(-q)$ are due to Jacobi, namely

$$
\varphi(q)=\prod_{n=1}^{\infty} \frac{\left(1-q^{2 n}\right)^{5}}{\left(1-q^{n}\right)^{2}\left(1-q^{4 n}\right)^{2}}, \quad \varphi(-q)=\prod_{n=1}^{\infty} \frac{\left(1-q^{n}\right)^{2}}{1-q^{2 n}} .
$$

Definition 3.1. For $k \in \mathbb{N}$ and $q \in \mathbb{C}$ with $|q|<1$, we define

$$
E_{k}=E_{k}(q):=\prod_{n=1}^{\infty}\left(1-q^{k n}\right)
$$


From (3.2) and Definition 3.1, we deduce

$$
\begin{aligned}
\varphi(q) & =E_{1}^{-2} E_{2}^{5} E_{4}^{-2}, \\
\varphi(-q) & =E_{1}^{2} E_{2}^{-1} .
\end{aligned}
$$

Lemma 3.1. For $k \in \mathbb{N}$ and $q \in \mathbb{C}$ with $|q|<1$,

$$
A_{k}(q)=\varphi^{8-k}(q) \varphi^{k}(-q) \text {. }
$$

Proof. We have

$$
\begin{aligned}
\prod_{n=1}^{\infty}\left(1+q^{n}\right) & =\prod_{n=1}^{\infty} \frac{1-q^{2 n}}{1-q^{n}}=E_{1}^{-1} E_{2}, \\
\prod_{n=1}^{\infty}\left(1-q^{4 n-2}\right) & =\prod_{n=1}^{\infty} \frac{\left(1-q^{4 n-2}\right)\left(1-q^{4 n}\right)}{1-q^{4 n}}=\prod_{n=1}^{\infty} \frac{1-q^{2 n}}{1-q^{4 n}}=E_{2} E_{4}^{-1} .
\end{aligned}
$$

Thus, by (1.6), we obtain

$$
\begin{aligned}
A_{k}(q) & =\prod_{n=1}^{\infty}\left(1+q^{n}\right)^{24-4 k}\left(1-q^{n}\right)^{8}\left(1-q^{4 n-2}\right)^{16-2 k}=E_{1}^{4 k-16} E_{2}^{40-6 k} E_{4}^{2 k-16} \\
& =\left(E_{1}^{-2} E_{2}^{5} E_{4}^{-2}\right)^{8-k}\left(E_{1}^{2} E_{2}^{-1}\right)^{k}=\varphi^{8-k}(q) \varphi^{k}(-q) . \text { - }
\end{aligned}
$$

Following Berndt [4, pp. 119-120] we set

$$
\begin{aligned}
& x=1-\frac{\varphi^{4}(-q)}{\varphi^{4}(q)}, \\
& z=\varphi^{2}(q) .
\end{aligned}
$$

From Berndt's catalogue of formulae for theta fuctions [4, p. 122] we have

$$
\begin{aligned}
\varphi(q) & =\sqrt{z}, \\
\varphi(-q) & =\sqrt{z}(1-x)^{1 / 4} .
\end{aligned}
$$

Following Cheng and Williams [5, p. 564] we set

$$
g=(1-x)^{1 / 4} .
$$

Lemma 3.2. For $k \in \mathbb{N}$ and $q \in \mathbb{C}$ with $|q|<1$, we have

$$
A_{k}(q)=g^{k} z^{4}
$$

Proof. By Lemma 3.1 and (3.7)-(3.9), we have

$$
A_{k}(q)=(\sqrt{z})^{8-k}\left(\sqrt{z}(1-x)^{1 / 4}\right)^{k}=(1-x)^{k / 4} z^{4}=g^{k} z^{4} .
$$

LEMMA 3.3.

$\sum_{n=1}^{\infty} c_{16}(n) q^{n}=\left(\frac{1}{32} g+\frac{3}{112} g^{2}+\frac{1}{224} g^{3}-\frac{1}{224} g^{5}-\frac{3}{112} g^{6}-\frac{1}{224} g^{7}\right) z^{4}$.

Proof. This follows from (1.5) and Lemma 3.2. 
Lemma 3.4. For $q \in \mathbb{C}$ with $|q|<1$, we have

$$
\sum_{\substack{n=1 \\ n \equiv 1(\bmod 4)}}^{\infty} c_{8}(n) q^{n}=\frac{1}{64}\left(g+2 g^{2}+g^{3}-g^{5}-2 g^{6}-g^{7}\right) z^{4} .
$$

(ii)

$$
\sum_{\substack{n=1 \\ n \equiv 3(\bmod 4)}}^{\infty} c_{8}(n) q^{n}=\frac{1}{64}\left(-g+2 g^{2}-g^{3}+g^{5}-2 g^{6}+g^{7}\right) z^{4} .
$$

(iii)

$$
\sum_{\substack{n=1 \\=0(\bmod 2)}}^{\infty} c_{8}(n / 2) q^{n}=\frac{1}{128}\left(g-g^{3}-g^{5}+g^{7}\right) z^{4} .
$$

Proof. Part (i) is [3, Theorem 2.3(i)]. Part (ii) is [3, Theorem 2.3(ii)]. By [3, Theorem 2.4] and (1.7), we have

$$
\sum_{\substack{n=1 \\ n \equiv 0(\bmod 2)}}^{\infty} c_{8}(n / 2) q^{n}=\sum_{\substack{n=1 \\ n \equiv 2(\bmod 4)}}^{\infty} c_{8}(n / 2) q^{n}=\frac{1}{128}\left(g-g^{3}-g^{5}+g^{7}\right) z^{4} .
$$

Theorem 3.1. For $n \in \mathbb{N}$,

$$
c_{16}(n)= \begin{cases}\frac{12}{7} c_{8}(n / 2) & \text { if } n \equiv 0(\bmod 2), \\ c_{8}(n) & \text { if } n \equiv 1(\bmod 4), \\ \frac{-1}{7} c_{8}(n) & \text { if } n \equiv 3(\bmod 4) .\end{cases}
$$

Proof. By Lemmas 3.3 and 3.4 we have

$$
\begin{aligned}
\sum_{n=1}^{\infty} c_{16}(n) q^{n}=\left(\frac{1}{32} g+\frac{3}{112} g^{2}+\frac{1}{224} g^{3}-\frac{1}{224} g^{5}-\frac{3}{112} g^{6}-\frac{1}{224} g^{7}\right) z^{4} \\
=\left(\frac{1}{64} g+\frac{1}{32} g^{2}+\frac{1}{64} g^{3}-\frac{1}{64} g^{5}-\frac{1}{32} g^{6}-\frac{1}{64} g^{7}\right) z^{4} \\
\quad-\frac{1}{7}\left(-\frac{1}{64} g+\frac{1}{32} g^{2}-\frac{1}{64} g^{3}+\frac{1}{64} g^{5}-\frac{1}{32} g^{6}+\frac{1}{64} g^{7}\right) z^{4} \\
\quad+\frac{12}{7}\left(\frac{1}{128} g-\frac{1}{128} g^{3}-\frac{1}{128} g^{5}+\frac{1}{128} g^{7}\right) z^{4} \\
=\sum_{n=1(\bmod 4)}^{\infty} c_{8}(n) q^{n}-\frac{1}{7} \sum_{n=1}^{\infty} c_{8}(n) q^{n}+\frac{12}{7} \sum_{n=1}^{\infty} c_{8}(n / 2) q^{n} .
\end{aligned}
$$

Equating coefficients of $q^{n}$, we obtain the assertion. 
We note that the first equality of Theorem 3.1 is Corollary 2.1 of [2]. We also observe that Theorem 3.1 can be expressed as

$$
c_{16}(n)=\frac{1}{7}\left(3+4\left(\frac{-4}{n}\right)\right) c_{8}(n)+\frac{12}{7} c_{8}(n / 2) .
$$

4. Proof of Theorem 1.1. We just prove part (i) as the remaining parts can be proved similarly. Appealing to (1.3), Proposition 1.1(i)-(ii), Definition 2.1 and Propositions 2.1-2.3, we obtain

$$
\begin{aligned}
& N\left(1^{7}, 4 ; n\right)=\sum_{m=0}^{n} N\left(1^{3}, 4 ; m\right) N\left(1^{4} ; n-m\right) \\
& =N\left(1^{4} ; n\right)+N\left(1^{3}, 4 ; n\right)+\sum_{m=1}^{n-1} N\left(1^{3}, 4 ; m\right) N\left(1^{4} ; n-m\right) \\
& =8 \sigma(n)-32 \sigma(n / 4) \\
& +\left(4+2\left(\frac{-4}{n}\right)\right) \sigma(n)-20 \sigma(n / 4)+24 \sigma(n / 8)-32 \sigma(n / 16) \\
& +\sum_{m=1}^{n-1}(4 \sigma(m)-20 \sigma(m / 4)+24 \sigma(m / 8)-32 \sigma(m / 16)) \\
& \times(8 \sigma(n-m)-32 \sigma((n-m) / 4)) \\
& +\sum_{m=1}^{n-1} 2\left(\frac{-4}{m}\right) \sigma(m)(8 \sigma(n-m)-32 \sigma((n-m) / 4)) \\
& =\left(12+2\left(\frac{-4}{m}\right)\right) \sigma(n)-52 \sigma(n / 4)+24 \sigma(n / 8)-32 \sigma(n / 16) \\
& +32 X(1,1 ; n)-160 X(4,1 ; n)+192 X(8,1 ; n)-256 X(16,1 ; n) \\
& -128 X(1,4 ; n)+640 X(4,4 ; n)-768 X(8,4 ; n) \\
& +1024 X(16,4 ; n)+16 \sum_{m=1}^{n-1}\left(\frac{-4}{m}\right) \sigma(m) \sigma(n-m) \\
& -64 \sum_{m=1}^{n-1}\left(\frac{-4}{m}\right) \sigma(m) \sigma((n-m) / 4) \\
& =\left(12+2\left(\frac{-4}{m}\right)\right) \sigma(n)-52 \sigma(n / 4)+24 \sigma(n / 8)-32 \sigma(n / 16) \\
& +32 W_{1}(n)-288 W_{4}(n)+192 W_{8}(n)-256 W_{16}(n)
\end{aligned}
$$




$$
\begin{aligned}
& +640 W_{1}(n / 4)-768 W_{2}(n / 4)+1024 W_{4}(n / 4) \\
& +16\left(\frac{-4}{n}\right)\left(\frac{1}{48} \sigma_{3}(n)+\left(\frac{1}{24}-\frac{n}{4}\right) \sigma(n)+\frac{3}{16} c_{8}(n)\right) \\
& +16 c_{8}(n / 2)-64\left(\frac{-4}{n}\right) W_{4}(n) .
\end{aligned}
$$

The asserted result now follows from Proposition 1.2 and Theorem 3.1.

\section{References}

[1] A. Alaca, Ş. Alaca, M. F. Lemire and K. S. Williams, Nineteen quaternary quadratic forms, Acta Arith. 130 (2007), 277-310.

[2] A. Alaca, Ş. Alaca and K. S. Williams, The convolution sum $\sum_{m<n / 16} \sigma(m) \times$ $\sigma(n-16 m)$, Canad. Math. Bull. 51 (2008), 3-14.

[3] - - - - Evaluation of the sums $\sum_{m=1, m \equiv a(\bmod 4)}^{n-1} \sigma(m) \sigma(n-m)$, Czechoslovak Math. J., to appear.

[4] B. C. Berndt, Number Theory in the Spirit of Ramanujan, Amer. Math. Soc., Providence, RI, 2006.

[5] N. Cheng and K. S. Williams, Convolution sums involving the divisor function, Proc. Edinburgh Math. Soc. 47 (2004), 561-572.

[6] J. G. Huard, Z. M. Ou, B. K. Spearman and K. S. Williams, Elementary evaluation of certain convolution sums involving divisor functions, in: Number Theory for the Millennium II, M. A. Bennett, B. C. Berndt, N. Boston, H. G. Diamond, A. J. Hildebrand and W. Philipp (eds.), A K Peters, Natick, 2002, 229-274.

[7] K. S. Williams, The convolution sum $\sum_{m<n / 8} \sigma(m) \sigma(n-8 m)$, Pacific J. Math. 228 (2006), 387-396.

Centre for Research in Algebra and Number Theory

School of Mathematics and Statistics

Carleton University

Ottawa, ON, Canada K1S 5B6

E-mail: aalaca@connect.carleton.ca salaca@connect.carleton.ca kwilliam@connect.carleton.ca 